\title{
Complications of Botulinum Toxin A: A Comprehensive Review
}

\author{
Steven H Dayan ${ }^{1,2,3}$, Christopher Ian Newberry ${ }^{4 *}$, Eric W Cerrati ${ }^{4}$ and Nazanin Ashourian ${ }^{1,3}$ \\ ${ }^{1}$ DeNova Research, Chicago, Illinois \\ ${ }^{2}$ Department of Otolaryngology, Division of Facial Plastic and Reconstructive Surgery, University of Illinois Medical Center, Chicago, Illinois \\ ${ }^{3}$ Chicago Center for Facial Plastic Surgery, Chicago, Illinois \\ ${ }^{4}$ Division of Otolaryngology-Head and Neck Surgery, University of Utah, UT, Austin
}

Received: 㘹 July 23, 2018; Published: 盏 July 30, 2018

*Corresponding author: Christopher Ian Newberry, Division of Otolaryngology-Head and Neck Surgery, University of Utah, Salt Lake City, UT, Austin

\begin{abstract}
Botulinum toxin was first described in the 1800's and has since found its permanent place in the medical community becoming the main ingredient of the most common cosmetic procedure. The injections have also gained multiple FDA approvals for both cosmetic and therapeutic indications. While the toxin injections have demonstrated a long history of safety, the drug remains highly controversial among regulators and legal entities. The following review provides a detailed, up-to-date description of botulinum toxin injection complications. Included topics are history, local and distal effects, long term safety, immunogenicity, use in pregnancy, and cosmetic use.
\end{abstract}

\section{Key Points}

a. Despite being one of the most studied pharmaceuticals and demonstrating a long history of safety, botulinum neurotoxin serotype A (BoNTA) remains to be a rather controversial drug and is under rigid scrutiny by the regulators and legal entities.

b. Given that botulinum toxin injections have become the most frequent cosmetic procedure, providers must be familiar with the product and the data outlining its safety profile.

\section{Introduction}

The first detailed record of botulism goes back to Justinus Kerner, a German poet and medical examiner, who linked the disease to the consumption of blood sausages and published detailed accounts of the associated symptoms along with the results of his experimental work on animals and himself [1]. Kerner recognized the causative agent as a "zoonic poison." It took another eighty years for Emile Pierre van Ermengem, professor of bacteriology at the University of Ghent, to identify the responsible pathogen as the anaerobic gram-positive bacterium Clostridium botulinum [1]. After its first identification, it wasn't for another 150 years for the botulinum neurotoxins (BoNTs) to be employed as therapeutics. In 1973, Alan Scott demonstrated that, in therapeutic doses, BoNTA can be used for alleviating the symptoms of strabismus [2]. In 1989, the

U.S. Food and Drug Administration (FDA) granted the first BoNTA approval for the treatment of blepharospasm and strabismus. Currently, the FDA has approved BoNTA for treating strabismus, blepharospasm, severe primary axillary hyperhidrosis, chronic migraine, cervical dystonia, overactive bladder, and glabellar as well as lateral canthal lines. The cosmetic benefits of BoNTA were illuminated in the 1980's through the important works of Drs. Alistair and Jean Carruthers [3]. In 2002, the FDA approved BoNTA for the temporary improvement of glabellar lines (frown lines) and in 2013 for the temporary reduction of moderate to severe lateral canthal lines (crow's feet) [4]. Today, with more than 6.6 million treatments annually, BoNTA is the most frequently performed non-surgical cosmetic procedure in the U.S [5]. Its popularity, nonetheless, is not without controversy. 
In 2009, the FDA required manufacturers to label all BoNTA products with a black box warning concerning the possible risk of adverse events (AEs) associated with the systemic spread of the toxin beyond the point of injection [6]. In the last two decades, numerous studies have established the safety and efficacy of BoNTA both in therapeutic and in cosmetic treatments [7-14]. The majority of reported AEs associated with BoNTA injections have been relatively mild and resolve without requiring any intervention. The most common AEs have involved injection site effects with the cosmetic use. Muscle paresis is an expected effect of BoNTA as the release of acetylcholine neurotransmitter is inhibited at the neuromuscular junctions near the site of injection, which may, consequently, lead to temporary local weakness. Thus, whereas nearly 30 years of clinical application and extensive research may seem to support the wide therapeutic index and safety profile of this product, concerns over undefined severe AEs and complications still exist. The most recent systematic review of the AEs reported to the FDA following a therapeutic or a cosmetic BoNTA treatment was performed in 2005 [9] reviewed all the AEs regarded as serious, per FDA regulations, from December 1989 to May 2003 as well as both the non-serious and serious AEs reported from December 2001 to November 2002. Among a total of 1,437 AEs reported to the FDA MedWatch system, 406 occurred after a therapeutic treatment and 1,031 after a cosmetic treatment. Of the 406 AEs reported following a therapeutic indication, 217 (53.4\%) were deemed serious and were associated with 28 deaths. It should, however, be noted that in 26 out of these 28 cases, the patients had an underlying disease that might have contributed to their death. Thirty-six serious AEs (3.49\%), but no deaths, were reported following the cosmetic use of BoNTA. Among these 36 reports, 13 patients had an underlying disease that might have contributed to the severity of the experienced AEs. Notably, of the 995 non-serious AEs, 623 (63\%) were reported as a lack of the intended cosmetic effect. Other common reported AEs were injection site reactions (19\%), ptosis (11\%), muscle weakness (5\%), and headache (5\%).

The results of [8] report must, however, be interpreted with caution as it contained multiple limitations, the most important of which is the lack of a proven causality between the reported AEs and the BoNTA injections. Not only may the reports not represent the accurate number of AEs observed in the population, but they may also be confounded by other influences such as publicity or a lack of proper medical understanding. Furthermore, patients who undergo any treatment, regardless of its type or medication, are more likely to notice even minor changes in their being and are more inclined to associate them with the treatment they had received. Thus, placebo-controlled studies are a much better method of monitoring AEs related to any treatment. Indeed, even though headaches constituted $5 \%$ of the reported AEs to the FDA MedWatch system, systematic review of clinical studies published from January 2000 to June 2012 determined that both the BoNTA treatment as well as the placebo group reported headaches and the difference between the two was not significant [15]. Therefore, the true number of treatment-related AEs may be lower than what is reported by [9].

\section{Distal effects of BoNTA}

Perhaps the greatest fear associated with the BoNTA treatment is that of contracting botulism. The spread of BoNTAs mainly occurs via local passive diffusion. While some suspect it may also spread via the blood or the nervous system, the clinical relevancy of this proposed long-distance migration is controversial. Whereas local diffusion can be expected to generate the same effects of denervation and weakness in muscles anatomically proximal to the site of injection, migration through the blood or the central nervous system (CNS) has been speculated to cause effects more distal to the injection site. There have been multiple reports of local diffusion of BoNTA from the injected to the adjacent muscles in animals. In 2008, Yaraskavitch et al. reported that BoNTA injections into the cat soleus muscle produces a significant loss of force in the target muscle and a smaller loss in the adjacent plantaris muscle [16]. It was concluded that the observed loss of force in the neighboring plantaris muscle is caused by the spread of toxin. It should, however, be noted that the ratio of injection volume to that of the muscle used in this study was higher than what is generally employed in clinical practice. Furthermore, only one dosage was tested and due to lack of reliable dose-response data for cat skeletal muscles, it is difficult to ascertain if the appropriate dose was used [17]. While the local diffusion of BoNTA is a likely phenomenon, as demonstrated in animal studies, its functional impact in clinical practice may not be significant. It is indeed possible that the dysphagia experienced by some patients following treatments with BoNTA for cervical dystonia or other movement disorders requiring injections into a patient's neck or head, is a consequence of local diffusion of the toxin to the pharyngeal musculature. Studies on BoNTA treatments in movement disorders, however, have established a pattern of long-term safety where the effectiveness of the treatments outweighs most observed AEs, which are generally infrequent and mild in nature $[8,12]$. In approved cosmetic applications, the most commonly concerning impact of local BoNTA dispersion can be expected to manifest as eyelid ptosis. The incident of reported eyelid ptosis, however, has been very low $[7,10,14]$, which may suggest local diffusion of BoNTA does not create significant clinical effects with the approved dosage in the cosmetic indications.

Additionally, the ptosis may be a result of a brow ptosis, more than the eyelid directly being affected, or a mild native ptosis being unmasked following the reduction in the frontalis muscle activity, which may be aiding in the lifting of the eyelids. Regardless of the etiology, diffusion can be a function of concentration and volume, and hence it is essential to administer the lowest dose needed in an appropriate volume to achieve the desired effects and avoid possible AEs. Distal effects of BoNTA have been confirmed only in its therapeutic indications. While systemic AEs have generally been rare and may include infrequent instances of flu-like symptoms and transient fatigue $[9,12]$, several cases of generalized muscle weakness have been reported. In 1997, Bakheit et al. first reported a systemic botulinum-like muscle weakness in two patients treated with the therapeutic doses of BoNTA [18]. The first patient, a 67-year-old woman who suffered from multiple 
sclerosis, was injected with $250 \mathrm{U}$ of abobotulinumtoxinA divided between the medial and lateral hamstring muscle of her left leg to relieve a severe spasticity. The second patient, a 34-year-old woman who suffered from multisystem atrophy, was injected with $250 \mathrm{U}$ of abobotulinumtoxinA in her right sternomastoid and left splenius capitis muscles as well as $\mathbf{5 0 0}$ units in her left midtrapezius. Both patients displayed systemic muscular weakness and distal denervation, determined by conventional intramuscular electromyography (EMG), consistent with the spread of toxin. All symptoms completely resolved after 4 weeks for the first patient and after 4 months for the second patient. Remarkably, whereas the symptoms of the first patient occurred 4 days after the first injection of BoNTA, the second patient had already been receiving regular treatments for 5 years before the onset of her systemic botulinumlike symptoms. Since the doses of BoNTA administered were below the maximum recommended for abobotulinumtoxin $A$ and the authors had prior evidence of use even up to 5,000 U without any associated AEs, an over-dose as the cause of the systemic symptoms was ruled out. Thus, due to the lack of any other obvious causes, it was suggested that some of the toxin might have inadvertently been injected into the blood vessels [18]. It is, however, important to note that there was no evidence directly supporting this assumption.

BoNTA was approved by the FDA for severe axillary hyperhidrosis in 2004. In double-blind clinical studies, in which 346 patients were exposed to $50 \mathrm{U}$ and 110 patients to $75 \mathrm{U}$ of onabotulinumtoxinA in each axilla, the most frequently reported AEs (3-10\% of adult patients) included injection site pain and hemorrhage, non-axillary sweating, infection, pharyngitis, flu syndrome, headache, fever, neck or back pain, pruritus, and anxiety. Furthermore, as discussed below, there are a multitude of reports in which doses even 10-times higher than $30 \mathrm{U}$ have been administered without complications. Thus, to our knowledge, the report of two transient cases of sub-acute botulism is the only incident in which BoNTA injections for palmar hyperhidrosis may have led to distal effects [19]. Due to the lack of any other evidence, human error cannot be ruled out, especially as both cases took place in the same medical center. It should also be noted that currently BoNTA is FDA approved only for severe axillary hyperhidrosis, and hence palmar applications are considered an off-label use. While there does not seem to be a pattern related to age, sex, site of injection, or dosage $(\mathrm{U} / \mathrm{kg})$, the majority of reported patients who suffered from a systemic botulinum-like symptoms received BoNTA injections for the treatment of spastic or dystonia disorders. It has been suggested that total units of BoNTA administered may be a more important factor than the dosage based on body weight $(\mathrm{U} / \mathrm{kg})$. It is important to account for the underlying neurological disorders of the patients and administer the lowest total dose that would provide relief while minimizing possible AEs. Multiple studies have attempted to determine the clinical manifestations of a distal spread of BoNTA. [20-22] performed six tests of cardiovascular autonomic reflexes and employed SF-EMG on the extensor digitorum communis muscle on five patients who were injected twice with increasing doses of Oculinum ${ }^{\circledR}$ for craniometrical dystonia and hemifacial spasm. Increased jitter values were detected in all five patients. Furthermore, it was reported that 4 out of 5 patients exhibited mild abnormalities in their cardiovascular reflexes. It was concluded that the BoNTA treatments affect the autonomic nervous system via a distant dispersion of the toxin [20]. Yet, remarkably, it was reported that all five patients had improvements in the symptoms of their underlying disorders following the BoNTA treatments without experiencing any AEs. Moreover, none of the patients displayed any symptoms that were associated with the observed increase in jitter values or the reported abnormalities in the cardiovascular reflexes. It should also be noted that it is difficult to establish causality between the subclinical observations and the BoNTA treatments since a control group was not utilized in the study.

Similar reports of systemic spread of BoNTA assessed by measuring distal neurotransmitter transmission [22] and changes in the autonomic system [23] are available. It is, however, not clear if the observed variations distant to the injection sites in patients treated with BoNTA for therapeutic indications have any significant clinical implications. Furthermore, no clear mechanisms for a systemic spread of BoNTA have been established. Retrograde axonal transport of BoNTA has been proposed as a possible route for the distal spread of the toxin to the CNS [24-29]. It should be noted that this proposed migration is mainly centered on basic scientific experimentations in animal models, and hence direct inferences to clinical applications must not be made. Spread of BoNTA to the CNS was first proposed in 1974. BoNTA inhibits the release of neurotransmitter acetylcholine by cleaving the SNAP-25 protein (synaptosomes-associated protein of $25 \mathrm{kDa}$ ) [30-33], which is a component of the SNARE complex in the cell membrane mediating the $\mathrm{Ca} 2+$-dependent exocytosis of synaptic vesicles [34-36]. To monitor the spread of BoNTA, [28] examined the distribution of cleaved SNAP-25 after injections in the central or peripheral targets in adult mice using a polyclonal antibody against BoNTA-cleaved SNAP-25. While the majority of BoNTA effects remained in the proximity of the injection sites, cleaved SNAP-25 was also observed in distant synapses. To examine the transport of BoNTA in motor neurons, a single dose of BoNTA was then injected into the whisker pad of adult rats. The facial nucleus ipsilateral to the injection site was dissected 3 days post-injection and the presence of cleaved SNAP-25 was assayed by immunoblotting. Cleaved SNAP-25 was observed in the facial nucleus and was also detected in the neutrophils surrounding the soma of facial motor neurons. The [28] report suffers from multiple limitations, and hence must be interpreted with caution. The BoNTA used in this study was prepared in the investigators' laboratory by a method described by Schiavo and Montecucco [28-37]. It is well-established that the different formulations of BoNTA employed in clinical applications, which are prepared and commercialized by various pharmaceutical companies, display distinct unit strengths and are not interchangeable [38]. Thus, direct comparisons between the biological potency of the BoNTA used in this study and the preparations for clinical applications cannot be made. 
Potential effects of BoNTA on CNS, whether direct or indirect, may have widespread applications in pain reducing treatments. In first reported a direct action of BoNTA on sensory neurons in vivo [39]. By utilizing a rat formalin inflammatory pain model, it was demonstrated that a single subcutaneous injection of BoNTA exerted analgesic effects without causing muscle weakness. It has been proposed that the antinociceptive effects of BoNTA are achieved by inhibiting the peripheral release of neurotransmitter glutamate, which reduces the release of neuropeptides, Substance $\mathrm{P}$ and calcitonin gene-related peptide, in the peripheral tissue, and decreases the transient receptor potential cation channel subfamily $\mathrm{V}$ member 1 trafficking to the peripheral neurons $[40,41]$. The effects of BoNTA in the treatment of nociceptive and pathological pain are active areas of research. In 2010, the FDA approved BoNTA for the prevention of headaches in adult patients with chronic migraine. Undoubtedly, examining the distal effects of BoNTA and especially its impact on CNS is of great interest as it has the potential to expand its therapeutic benefits. Despite the reports from animal model studies, the significance of a distal spread of BoNTA, possibly through the proposed axonal retrograde or a hematogenous transport, remains unclear in clinical practice. Unfortunately, the results from some reports have been overinterpreted and inaccurately extrapolated to clinical applications.

\section{Long-term safety of BoNTA}

Numerous reports examining the long-term effects of treatment with BoNTA has already been published [7,8,11,12,14,42,43] In a 2013 article describing an assessment of at least 10 years of consecutive treatments with BoNTA for blepharospasm and hemifacial spasm, Ababneh et al. confirmed the results of previous reports on the long-term safety and efficacy of BoNTA therapy for focal dystonia [42-47]. AEs had occurred in $11.1 \%$ and $3.8 \%$ of all BoNTA treatments in the first and last years, respectively, and common events included ptosis, dry eye, and lagophthalmos. Furthermore, none of the AEs were reported as life threatening or resulted in the discontinuation of the therapy. The success rate, defined as the patients' self-assessment of a good or excellent response, was reported at $93.8 \%$ in the first year and $96.9 \%$ in the last year (mean follow-up period of 14.1 years and maximum of 20 years). It was, however, noted that to maintain efficacy, the mean dosage had increased significantly from $22.5 \pm 7.5 \mathrm{U}$ of onabotulinumtoxinA during the first year to $26.8 \pm 10.3 \mathrm{U}$ after more than 10 years of continuous therapy. The increase in dosage was attributed to the chronic, progressive nature of focal dystonia as well as perhaps the formation of low titer neutralizing antibodies during the long course of treatments [42]. It is interesting to note that the increase in the therapeutic dosage, however, coincided with a reduction in the number of observed AEs with continued therapy. [8] performed a retrospective study of 235 patients receiving a total of 2,616 BoNTA applications for movement disorders, including cervical dystonia, hemifacial spasm, and blepharospasm, in a period of over 10 years. Observed AEs were generally mild and occurred in $26.9 \%$ of patients at any one time over $4.5 \%$ of the total applications. It was reported that only $1.3 \%$ of patients had discontinued the treatment due to the associated AEs, including 3 patients with cervical dystonia who experienced dysphagia in the first year of treatment. As elucidated above, this is in agreement with other reports indicating that while the efficacy of BoNTA treatments remains unchanged or even increases, the rate of observed AEs declines with long-term continuous therapy.

Long-term cosmetic applications of BoNTA have also established a record of safety and efficacy. Recently, Trindade de Almeida et al. examined data from 194 patients who had received a total of 4,402 treatments for glabellar lines over a mean of 9.1 years [7]. The mean number of injections was $22.7 \pm 8.7$ with a mean dose of $21.6 \pm 10.8 \mathrm{U}$ of onabotulinumtoxinA per treatment. Among the patients, $92.3 \%$ had also received treatments for latheral canthal lines (mean dose of $19.4 \pm 8.6 \mathrm{U}$ ), $90.7 \%$ for forehead lines $(9.0 \pm$ $5.6 \mathrm{U}), 51.5 \%$ for chin $(6.6 \pm 2.4 \mathrm{U}), 34.5 \%$ for perioral lines $(4.1 \pm$ $1.5 \mathrm{U})$, and $33 \%$ for bunny lines $(6.3 \pm 2.4 \mathrm{U})$. Reported AEs were infrequent (0.015 AEs per application), mostly mild in severity, and declined in frequency with continuous treatment. Furthermore, it was reported that $92.3 \%$ of patients were mostly or very satisfied with the results and $89.7 \%$ reported looking younger. Thus, in over 26 years of clinical use, since first approved by the FDA in 1989 for strabismus and blepharospasm, BoNTA has established a record of long-term safety and efficacy both for therapeutic as well as the cosmetic indications.

\section{Immunogenicity of BoNTA}

BoNTA is produced as a complex of multiple proteins (neurotoxin-associated proteins, NAPs) that protect the active 150 KDa unit from unfavorable environmental conditions $[31,48,49]$. As the toxin is a foreign agent to the human body and is supplied as a large complex, it can induce the host immune response that may inactivate the toxin. Furthermore, the clinical indications of BoNTA are generally for chronic disorders that require life-long treatments. The therapeutic effects of BoNTA are also transient, and, thus, recurrent administration is required to sustain the benefits. Longterm regular applications have been hypothesized to contribute to the production of antibodies against the toxin. Efficacy failure as a result of neutralizing antibodies, is considered a secondary failure as it occurs during the course of an ongoing therapy when prior treatments had produced effective outcomes [50]. It should, however, be noted that not all secondary failures can be attributed to the production of neutralizing antibodies by the host. Worsening of the underlying disease and subjective patient-related factors such as expectations, a placebo effect of the prior applications, or receiving a repeat treatment before a return to the baseline can all lead to secondary treatment failures [51]. Additionally, not all antibodies that are produced against the neurotoxin complex have an inactivating effect. BoNTA is a complex of a core neurotoxin with up to 6 NAPs, which mainly function to protect the active unit from harmful environmental factors such as the acidic condition of the host gastrointestinal tract $[31,48,49]$. It has been illustrated that in physiological conditions, NAPs dissociate from the active core. This dissociation is believed to occur upon reconstitution of the toxin 
with a saline solution in vitro, and hence even before injection [48]. Therefore, antibodies produced against the NAPs are less likely to inhibit the active core of the toxin, and, thus, can be considered mostly as non-neutralizing. Yet, NAPs may potentially increase the immunogenic risk of the neutralizing antibody formation since they increase the foreign protein load in the host. In agreement with this premise, the number of non-responders has declined since a new formulation of onabotulinumtoxinA was introduced in 1999 that significantly reduced the total size of the complex [52].

The reported prevalence of BoNTA immune resistance varies in the literature. The values may depend on a multitude of variables such as the indication, dosage, frequency of administration, formulation and manufacturing process, and the study design (for a detailed review see [53]). It is generally thought that the higher dosage and more frequent injections along with a greater volume of excipient proteins may contribute significantly to the development of antibodies against BoNTA. Remarkably, the detection of neutralizing antibodies by different laboratory tests does not equate to the inactivation of the toxin and a nonresponsive therapy [54,55]. For example, in the Naumann et al. meta-analysis study, only 3 out of 11 subjects who had developed neutralizing antibodies were reported to be clinically unresponsive to BoNTA treatments, [53] which corresponds to only $0.13 \%$ of total patients analyzed $(2,240)$. Therefore, the prevalence of neutralizing antibody formation seems to be very low with BoNTA treatments, and while neutralizing antibodies may be detected by different laboratory tests, the clinical implications remain unclear.

\section{BoNTA in pregnancy}

The data regarding the effects of BoNTA in pregnancy is scarce. As a result, BoNTA is considered a Category $\mathrm{C}$ drug by the FDA indicating that it should be used only if the potential benefits would Table 1: Impact of onabotulinumtoxinA in pregnant animals. justify the potential risks to the fetus. Consequently, the standard of care in the U.S. is to avoid treating patients who are pregnant or are attempting to get pregnant with BoNTA, especially for the cosmetic indications. Hilde band et al. measured the concentration of BoNTA in different body fluids of pregnant rabbits that were injected intravenously with a lethal dose of the toxin [56]. There were no detectable levels of BoNTA present in the placenta or the fetal blood at the time of the rabbits' death. Furthermore, molecules greater than $500 \mathrm{Da}$ are thought to exhibit an incomplete transfer through the human placenta [57]. As the catalytically active form of BoNTA is about $150 \mathrm{kDa}$, it is unlikely that BoNTA would diffuse through the human placenta. An active transport mechanism, however, cannot be ruled out. Effects of BoNTA administration with various doses and schedules have been examined in animals using onabutulinmtoxinA (Table 1) and abobotulinumtoxinA (Table 2). Based on these studies, the no-effect dose for a single maternal injection of onabotulinumtoxinA in rats $(16 \mathrm{U} / \mathrm{kg})$ is approximately 50 times greater than the average cosmetic dosage generally administered in humans. There have, however, been no formal studies examining the effects of BoNTA administration in pregnant woman, and the available evidence is mainly from various case studies [58,59], first reported the case of a 26-year-old woman who experienced four apparently uncomplicated pregnancies while receiving regular treatments with $300 \mathrm{U}$ of onabotulinumtoxinA for idiopathic cervical dystonia. The patient had received a $300 \mathrm{U}$ dose in the 3-month period before all 4 pregnancies and received 3 additional treatments in 1 pregnancy and 2 additional treatments in the other pregnancies for a total dosage up to $1200 \mathrm{U}$ in each pregnancy. None of the children were breast-fed. While the children were not assessed directly, there were no suggestions of cognitive or motor developmental delays during the follow-up period, which was about 5 years for the oldest child [58].

\begin{tabular}{|c|c|c|c|c|}
\hline Animal Model & Dose & $\begin{array}{c}\text { Administration } \\
\text { Schedule }\end{array}$ & Developmental Stage & Effect \\
\hline Mice, Rats & $4 \mathrm{U} / \mathrm{Kg}$ & $2 \mathrm{X}$ daily & Gestation days 5 and 13 & No effect \\
\hline Rats, Rabbits & $1 \mathrm{U} / \mathrm{Kg}$ (rats), $0.25 \mathrm{U} / \mathrm{Kg}$ (rabbits) & $1 \mathrm{X}$ daily & Gestation period & No effect \\
\hline Rats, Rabbits & $\geq 4 \mathrm{U} / \mathrm{Kg}$ (rats), $\geq 8 \mathrm{U} / \mathrm{Kg}$ (rabbits) & $1 \mathrm{X}$ daily & Gestation period & Maternal toxicity \\
\hline Rats & 1,4, or $16 \mathrm{U} / \mathrm{Kg}$ & $1 \mathrm{X}$ & $\begin{array}{c}\text { Prior to implantation, implantation, } \\
\text { or organogenesis }\end{array}$ & No effect \\
\hline
\end{tabular}

Data from onabotulinumtoxinA prescribing information, Allergan, Inc [33].

Table 2: Impact of abobotulinumtoxinA in pregnant animals.

\begin{tabular}{|c|c|c|c|c|}
\hline Animal Model & Dose & $\begin{array}{c}\text { Administration } \\
\text { Schedule }\end{array}$ & Developmental Stage & Effect \\
\hline Rats & $2.2,6.6,22 \mathrm{U} / \mathrm{Kg}$ & $1 \mathrm{X}$ daily & $\begin{array}{c}\text { From gestation days } 6 \text { through } \\
17\end{array}$ & Early embryonic death \\
\hline Rats & $44 \mathrm{U} / \mathrm{Kg}$ & $1 \mathrm{X}$ & Gestation day 6 and 12 & Early embryonic death \\
\hline Rats & $22.2 \mathrm{U} / \mathrm{Kg}$ & $1 \mathrm{X}$ weekly & $\begin{array}{c}\text { Gestation day } 6 \text { through } \\
\text { parturition to weaning }\end{array}$ & No effect \\
\hline
\end{tabular}

Data from abobotulinumtoxinA prescribing information, Galderma Laboratories. 
There have also been reports of women who contracted foodborne botulism during pregnancy $[59,60]$ have described a 37-year-old woman who was admitted to the hospital at week 23 of gestation for the suspicion of contracting botulism. The patient developed symptoms of muscle weakness, dysarthria, dysphagia, and tetraparesis requiring respiratory support for 2 months before recovering. She was discharged from the hospital 3 months after the onset of symptoms. Remarkably, it was noted that at the most intense point of the disease, when the patient's muscles were almost completely paralyzed, fetal movements were the only visible motion. Moreover, there were no detrimental effects on fetal development, and the patient had a normal spontaneous delivery one month after her discharge from the hospital [59]. To determine the physicians' experience with BoNTA administration during pregnancy, Morgan et al. surveyed 900 physicians who used commercially available BoNTA in their practice. Among the $396(44 \%)$ of the questionnaires returned, 12 physicians $(3 \%$ of responders) had injected pregnant woman with BoNTA. Of the 19 total pregnancies, one was terminated medically, and one was miscarried. The patient who miscarried was injected with $300 \mathrm{U}$ of BoNTA for cervical dystonia and had a history of spontaneous abortion; therefore, a causal relationship between her miscarriage and the BoNTA injections could not be established. The other 17 pregnancies were reported to have gone to full term without complications and without any special postnatal care for the infants [61]. Based on the limited information currently available, administration of BoNTA, at least with doses at $300 \mathrm{U}$ or less, does not seem to cause complications in pregnancy or harm the fetus. It would be a reasonable estimate that approximately 10 million injections annually are performed in women who are, at least unknowingly, pregnant. Currently many of the childbearing women who receive therapeutic treatments for disorders such as cervical dystonia, have to choose between continuing the benefits of their therapy and becoming pregnant.

\section{Cosmetic BoNTA and safety}

The dose of BoNTA used in cosmetic procedures (20 U onabotulinumtoxinA or $85 \mathrm{U}$ abobotulinumtoxinA for glabellar lines and $24 \mathrm{U}$ onabotulinumtoxinA for lateral canthal lines) is a few folds lower than what is commonly administered for the therapeutic indications. Thus, it may be expected that the rate of treatmentrelated AEs would be even lower in the cosmetic applications. In a recent report, Cavallini et al. performed a systematic review of clinical studies published from January 2000 to June 2012 in which BoNTA (60\% onabotulinumtoxinA, 37.1\% abobotulinumtoxinA, and $2.8 \%$ incobotulintoxinA) was used for cosmetic procedures and confirmed the short-term safety for all its three formulations [15]. Of a total of 8,787 patients, $51.4 \%$ had received treatments for the glabellar lines, $25.7 \%$ for the upper face, $11.4 \%$ for the lateral canthal lines, and $11.4 \%$ for the lower face. All treatment-related AEs, which included blepharoptosis (2.5\%), brow ptosis $(3.1 \%)$, eye sensory disorders (3\%), and lip asymmetries and imbalances in the lower face (6.9\%), resolved spontaneously. In another metaanalysis, Brin et al. examined data from a total of 1,678 patients from 6 randomized, double-blind placebo-controlled and 3 openlabel studies for glabellar and lateral canthal lines [14]. The overall rate of treatment-related AEs was reported at $24 \%$ in the treatment group and $16 \%$ in the placebo group $(\mathrm{p}<0.01)$. Eyelid ptosis, eyelid sensory disorder, and eyelid edema were the only 3 AEs that had a significantly higher incident in the treatment group as compared with the placebo group. Moreover, no systemic weakness or distal nervous system effects were reported.

The long-term safety of cosmetic BoNTA has also been established over the past decade since it was first approved for glabellar lines in 2002. As discussed earlier, in Trindade de Almeida et al examination of 194 patients who received a total of 4,402 treatments over a mean of 9.1 years, the incident of AEs was 0.015 per application. The reported AEs were mild in severity and similar to the therapeutic indications. They also declined in frequency with continuous treatment [7]. Thus, many years of clinical practice have provided convincing evidence for both short- and long-term safety and high therapeutic index of BoNTA in cosmetic procedures.

\section{Concluding remarks}

BoNTA, one of the world's most potent toxins, has been revealed to provide a broad range of therapeutic as well aesthetic benefits. Despite being one of the most studied pharmaceuticals and demonstrating a long history of safety with widespread use, BoNTA remains a controversial drug and is under a rigid scrutiny by the regulators and legal entities. Its use continues to grow in popularity for both on and off-label cosmetic indications making up $47 \%$ of all minimally-invasive cosmetic procedures in 2013. This number has increased by $703 \%$ in just over a decade [62]. Even though over two decades of clinical experience provides support for the efficacy and safety of BoNTA, it is incumbent that we, as physicians, always listen and evaluate whether any medications, from aspirin to ibuprofen to BoNTA, provides the desired clinical outcomes as well as any discomforts or adverse events for our patients. It is also necessary that we use facts based on scientific and clinical research to educate our patients as well as those who are responsible for making decisions in the risks and benefits any medication would pose to the public and to the patients.

\section{References}

1. Erbguth FJ (2004) Historical notes on botulism, Clostridium botulinum, botulinum toxin, and the idea of the therapeutic use of the toxin. Mov Disord 19(S8): S2-S6.

2. Scott $A B$ (1980) Botulinum toxin injection into extraocular muscles as an alternative to strabismus surgery. Ophthalmology 87(10): 1044-1049.

3. Carruthers JD, Carruthers JA (1992) Treatment of glabellar frown lines with C. botulinum-A exotoxin. J Dermatol Surg Oncol 18(1): 17-21.

4. (2013) FDA Press Announcements FDA approves Botox Cosmetic to improve the appearance of crow's feet lines p. 1-3.

5. (2015) ASPS Cosmetic Plastic Surgery Statistics.

6. Kuehn BM (2009) FDA Requires Black Box Warnings on Labeling for Botulinum Toxin Products. JAMA 301(22): 2316-2316.

7. Trindade de Almeida A, Carruthers J, Cox SE, Goldman MP, Wheeler S, et al. (2015) Patient satisfaction and safety with aesthetic onabotulinumtoxinA 
after at least 5 years: a retrospective cross-sectional analysis of 4,402 glabellar treatments. Dermatol Surg 41(Suppl1): S19-S28.

8. Hsiung GYR, Das SK, Ranawaya R, Lafontaine AL, Suchowersky O (2002) Long-term efficacy of botulinum toxin A in treatment of various movement disorders over a 10-year period. Mov Disord 17(6): 12881293

9. Coté TR, Mohan AK, Polder JA, Walton MK, Braun MM (2005) Botulinum toxin type A injections: Adverse events reported to the US Food and Drug Administration in therapeutic and cosmetic cases. Journal of the American Academy of Dermatology 53(3): 407-415.

10. Moy R, Maas C, Monheit G, Huber MB, Reloxin (2009) Investigational Group. Long-term safety and efficacy of a new botulinum toxin type A in treating glabellar lines. Arch Facial Plast Surg 11(2): 77-83.

11. Niamtu J (2003) Botulinum toxin A: A review of 1,085 oral and maxillofacial patient treatments. Journal of Oral and Maxillofacial Surgery 61(3): 317-324.

12. Ramirez Castaneda J, Jankovic J (2013) Long-Term Efficacy and Safety of Botulinum Toxin Injections in Dystonia. Toxins 5(2): 249-266.

13. Dailey RA, Philip A, Tardie G (2011) Long-term treatment of glabellar rhytides using onabotulinumtoxina. Dermatologic Surgery 37(7): 918928.

14. Brin MF, Boodhoo TI, Pogoda JM (2009) Safety and tolerability of onabotulinumtoxinA in the treatment of facial lines: a meta-analysis of individual patient data from global clinical registration studies in 1678 participants. Journal of the American Academy of Dermatology 61(6): 961-970.

15. Cavallini M, Cirillo P, Fundarò SP (2014) Safety of Botulinum Toxin A in Aesthetic Treatments: A Systematic Review of Clinical Studies. Dermatologic Surgery 40(5): 525-536.

16. Yaraskavitch M, Leonard T, Herzog W (2008) Botox produces functional weakness in non-injected muscles adjacent to the target muscle. Journal of Biomechanics 41(4): 897-902.

17. Aoki KR, Irvine R, Gallagher C (2008) Re: Botox produces functiona weakness in non-injected muscles adjacent to the target muscle. Journal of Biomechanics, author reply 2067 41(9): 2066-2067.

18. Bakheit AM, Ward CD, McLellan DL (1997) Generalised botulism-like syndrome after intramuscular injections of botulinum toxin type A: a report of two cases. J Neurol Neurosurg Psychiatr 62(2): 198.

19. Ghasemi M, Norouzi R, Salari M, Asadi B (2012) Iatrogenic Botulism After the Therapeutic Use of Botulinum Toxin-A. Clin Neuropharmacol 35(5): 254-257.

20. Girlanda P, Vita G, Nicolosi C, Milone S, Messina C (1992) Botulinum toxin therapy: distant effects on neuromuscular transmission and autonomic nervous system. J Neurol Neurosurg Psychiatr 55(9): 844-845.

21. Lange DJ, Rubin M, Greene PE (1991) Distant effects of locally injected botulinum toxin: a double-blind study of single fiber EMG changes. Muscle Nerve 14(7): 672-675.

22. Lange DJ, Brin MF, Warner CL, Fahn S, Lovelace RE (1987) Distant effects of local injection of botulinum toxin. Muscle Nerve 10(6): 552-555.

23. Tintner R, Gross R, Winzer UF, Smalky KA, Jankovic J (2005) Autonomic function after botulinum toxin type A or B: a double-blind, randomized trial. Neurology 65(5): 765-767.

24. Habermann E (1974) 125I-labeled neurotoxin from Clostridium botulinum A: preparation, binding to synaptosomes and ascent to the spinal cord. Naunyn Schmiedebergs Arch Pharmacol 281(1): 47-56.

25. Wiegand H, Erdmann G, Wellhöner HH (1976) 125I-labelled botulinum A neurotoxin: pharmacokinetics in cats after intramuscular injection. Naunyn Schmiedebergs Arch Pharmacol 292(2): 161-165.

26. Black JD, Dolly JO (1986) Interaction of 125I-labeled botulinum neurotoxins with nerve terminals. I. Ultrastructural autoradiographic localization and quantitation of distinct membrane acceptors for types A and B on motor nerves. Journal of Cell Biology 103(2): 521-534.

27. Hagenah R, Benecke R, Wiegand H (1977) Effects of type A botulinum toxin on the cholinergic transmission at spinal Renshaw cells and on the inhibitory action at IA inhibitory interneurones. Naunyn Schmiedebergs Arch Pharmacol 299(3): 267-272

28. Antonucci F, Rossi C, Gianfranceschi L, Rossetto O, Caleo M (2008) LongDistance Retrograde Effects of Botulinum Neurotoxin A. Journal of Neuroscience 28(14): 3689-3696.

29. Restani L, Antonucci F, Gianfranceschi L, Rossi C, Rossetto O, et al. (2011) Evidence for anterograde transport and transcytosis of botulinum neurotoxin A (BoNT/A). J Neurosci 31(44): 15650-15659.

30. Wheeler A, Smith HS (2013) Botulinum toxins: Mechanisms of action, antinociception and clinical applications. Toxicology 306: 124-146.

31. Tighe AP, Schiavo G (2013) Botulinum neurotoxins: Mechanism of action. Toxicon 67: 87-93.

32. Blasi J, Chapman ER, Link E (1993) Botulinum neurotoxin A selectively cleaves the synaptic protein SNAP-25. Nature 365(6442): 160-163.

33. Keller JE (2001) The Role of the Synaptic Protein SNAP-25 in the Potency of Botulinum Neurotoxin Type A. Journal of Biological Chemistry 276(16): 13476-13482.

34. Söllner TH (2003) Regulated exocytosis and SNARE function (Review). Mol Membr Biol 20(3): 209-220.

35. Zhang X, Kim-Miller MJ, Fukuda M, Kowalchyk JA, Martin TFJ (2002) Ca2+-Dependent Synaptotagmin Binding to SNAP-25 Is Essential for Ca2+-Triggered Exocytosis. Neuron 34(4): 599-611.

36. Söllner T, Whiteheart SW, Brunner M (1993) SNAP receptors implicated in vesicle targeting and fusion. Nature 362(6418): 318-324.

37. Schiavo G, Montecucco C (1995) Tetanus and botulism neurotoxins: isolation and assay. Methods in Enzymology 248: 643-652.

38. Walker T], Dayan SH (2014) Comparison and overview of currently available neurotoxins. J Clin Aesthet Dermatol 7(2): 31-39.

39. Cui M, Khanijou S, Rubino J, Aoki KR (2004) Subcutaneous administration of botulinum toxin A reduces formalin-induced pain. Pain 107(1): 125133.

40. Francisco GE, Tan H, Green M (2012) Do Botulinum Toxins Have a Role in the Management of Neuropathic Pain? American Journal of Physical Medicine \& Rehabilitation 91(10): 899-909.

41. Pavone F, Luvisetto S (2010) Botulinum Neurotoxin for Pain Management: Insights from Animal Models. Toxins 2(12): 2890-2913.

42. Ababneh OH, Cetinkaya A, Kulwin DR (2013) Long-term efficacy and safety of botulinum toxin A injections to treat blepharospasm and hemifacial spasm. Clin Experiment Ophthalmol 42(3): 254-261.

43. Bentivoglio AR, Fasano A, Ialongo T, Soleti F, Fermo Lo S, et al. (2009) Fifteen-Year Experience in Treating Blepharospasm with Botox or Dysport: Same Toxin, Two Drugs. Neurotox Res 15(3): 224-231.

44. Gill HS, Kraft SP (2010) Long-term efficacy of botulinum a toxin for blepharospasm and hemifacial spasm. Can J Neurol Sci 37(5): 631-636.

45. Naumann M, Albanese A, Heinen F, Molenaers G, Relja M (2006) Safety and efficacy of botulinum toxin type $A$ following long-term use. European Journal of Neurology 13(Suppl 4): 35-40.

46. Mejia NI, Vuong KD, Jankovic J (2005) Long-term botulinum toxin efficacy, safety, and immunogenicity. Mov Disord 20(5): 592-597.

47. Czyz CN, Burns JA, Petrie TP, Watkins JR, Cahill KV, et al. (2013) Longterm botulinum toxin treatment of benign essential blepharospasm, hemifacial spasm, and Meige syndrome. Am J Ophthalmol 156(1): 173177.

48. Eisele KH, Fink K, Vey M, Taylor HV (2011) Studies on the dissociation of botulinum neurotoxin type A complexes. Toxicon 57(4): 555-565. 
49. Rossetto O, Pirazzini M, Montecucco C (2014) Botulinum neurotoxins: genetic, structural and mechanistic insights. Nature Publishing Group 12(8): 535-549.

50. Dressler D (2004) Clinical presentation and management of antibodyinduced failure of botulinum toxin therapy. Mov Disord 19(Suppl 8): S92-S100.

51. Benecke R (2012) Clinical relevance of botulinum toxin immunogenicity. Bio Drugs 26(2): e1-e9.

52. Aoki KR, Garrett M, Spanoyannis A, Wheeler L (1999) BOTOX® (Botulinum Toxin Type A) Purified Neurotoxin Complex Prepared from the New Bulk Toxin Retains the Same Preclinical Efficacy as the Original but with Reduced Immunogenicity. Neurology 52(6, Suppl 2): A521-A522.

53. Naumann M, Boo LM, Ackerman AH, Gallagher CJ (2013) Immunogenicity of botulinum toxins. J Neural Transm 120(2): 275-290.

54. Kranz G, Sycha T, Voller B, Kranz GS, Schnider P, et al. (2008) Neutralizing antibodies in dystonic patients who still respond well to botulinum toxin type A. Neurology 70(2): 133-136.
55. Lange O, Bigalke H, Dengler R, Wegner F, DeGroot M, et al. (2009) Neutralizing antibodies and secondary therapy failure after treatment with botulinum toxin type A: much ado about nothing? Clin Neuropharmacol 32(4): 213-218.

56. Hildebrand GJ, Lamanna C, Heckly RJ (1961) Distribution and Particle Size of Type A Botulinum Toxin in Body Fluids of Intravenously Injected Rabbits. Exp Biol Med 107(2): 284-289.

57. Pacifici GM, Nottoli R (1995) Placental transfer of drugs administered to the mother. Clin Pharmacokinet 28(3): 235-269.

58. Newman WJ, Davis TL, Padaliya BB (2004) Botulinum toxin type A therapy during pregnancy. Mov Disord 19(11): 1384-1385.

59. Polo JM, Martin J, Berciano J (1996) Botulism and pregnancy. Lancet 348(9021): 195.

60. St Clair EH, DiLiberti JH, O Brien ML (1975) Letter: Observations of an infant born to a mother with botulism. J Pediatr 87(4): 658.

61. Morgan JC (2006) Botulinum toxin A during pregnancy: a survey of treating physicians. J Neurol Neurosurg Psychiatr 77(1): 117-119.

62. (2014) ASPS Plastic Surgery Statistics Report p. 1-23.

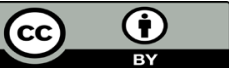

This work is licensed under Creative Commons Attribution 4.0 License

To Submit Your Article Click Here:

Submit Article
DOI: $10.32474 /$ SJ0.2018.01.000105

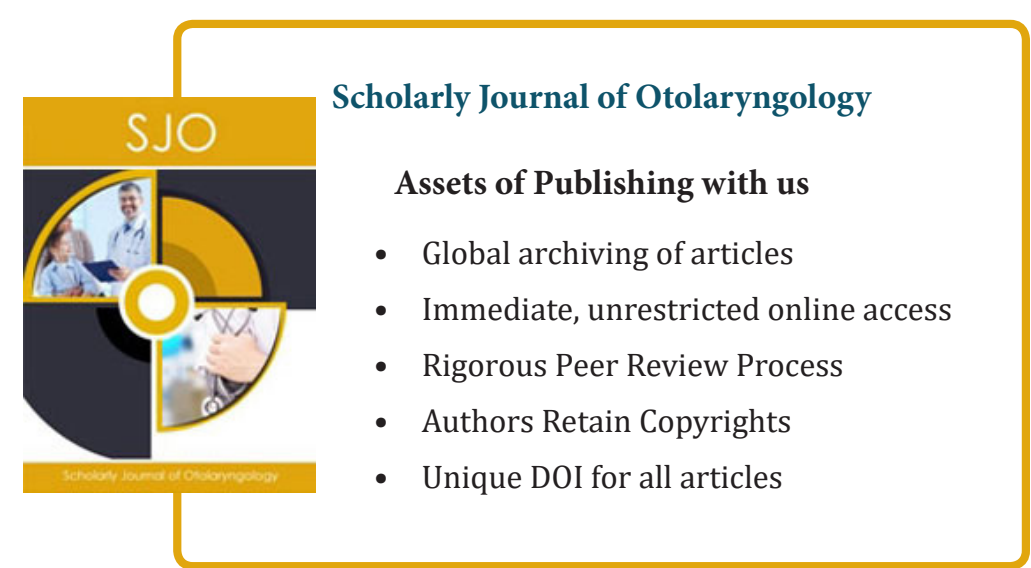

